PRIMER NOTE

\title{
Isolation and characterization of 15 microsatellite loci in the poplar rust fungus, Melampsora larici-populina, and cross-amplification in related species
}

\author{
BENOÎT BARRÈS, ${ }^{*}$ CYRIL DUTECH, + AXELLE ANDRIEUX,${ }^{*}$ HENRI CARON,$+J E A N ~ P I N O N *$ and \\ PASCAL FREY* \\ *UMR IAM, Pathologie Forestière, INRA Nancy, 54280 Champenoux, France, +UMR BIOGECO, INRA Pierroton, 33612 Cestas, France
}

\begin{abstract}
We developed 15 microsatellite loci in the poplar rust fungus, Melampsora larici-populina, using two enrichment protocols. Polymorphism of each locus was assessed on a panel of 30 isolates, comprising three subpanels (world, regional and local scales). Thirteen loci were polymorphic with three to eight alleles detected. The 15 loci were also tested on five related Melampsora species, M. allii-populina, M. medusae f. sp. deltoidae, M. larici-tremulae, $M$. rostrupii and $M$. pinitorqua, and partial or global cross-amplification events were detected.
\end{abstract}

Keywords: Basidiomycete, cross-species amplification, enriched library, Melampsora larici-populina, microsatellites

Received 24 June 2005; revision received 8 July 2005; accepted 18 July 2005

The Basidiomycete Melampsora larici-populina causes foliar rust on Populus species from the sections Aigeiros and Tacamahaca and their hybrids (Frey et al. 2005). During the last few decades, this pathogen has caused important economic losses to poplar cultivation in Europe - mainly based on Populus $\times$ euramericana and Populus $\times$ interamericana hybrids - due to the breakdown of several major resistance genes (Pinon \& Frey 2005). Random amplified polymorphic DNA markers were developed for M. larici-populina to compare genetic diversity between strains occurring on cultivated hybrid poplar stands and wild riparian Populus nigra stands (Frey et al. 2005). However, observed heterozygosity cannot be detected in this dicaryotic fungus with these dominant markers, which prompted a search for codominant microsatellite markers. Microsatellite markers have already been developed on several Basidiomycetes including rust fungi (e.g. Enjalbert et al. 2002).

Since microsatellites seem to be less frequent in fungal genomes than in other organisms (Lim et al. 2004), the strategy of isolating loci in M. larici-populina was to build an

Correspondence: P. Frey, Fax: +33383394069;

E-mail: frey@nancy.inra.fr enriched library for microsatellites. Total genomic DNA was extracted from M. larici-populina isolates 93ID6 and 98AG31 (Table 1) using DNeasy Plant Mini Kit (QIAGEN) after grinding $5 \mathrm{mg}$ of urediniospores with glass beads (Pei et al. 1997). A first library was constructed according to Edwards et al. (1996) using Hybond membranes with two hybridization rounds. A second enriched library using biotinylated oligoprobes and streptavidin-coated magnetic beads was constructed following the protocol of Dutech et al. (2000) to increase the number of loci. Both libraries were enriched with (TC) ${ }_{10}$ and (TG) ${ }_{10}$ motifs and cloned using a TOPO TA Cloning Kit (Invitrogen). In the second library, a colony blot screening according to Estoup et al. (1993) and a polymerase chain reaction (PCR) size screening using vector's primers were performed to select clones larger than $500 \mathrm{bp}$ and containing microsatellite motifs. In the first library, 104 clones were selected and sequenced, and 20 contained a microsatellite motif larger than $20 \mathrm{bp}$ in length. In the second library, 3930 clones were screened and 978 gave a positive response for microsatellite presence. After size screening, 96 clones were sequenced and 17 were chosen for primer design. Primers were designed using PRIMER 3 software (Rozen \& Skaletsky 2000) and tested 
PRIMER NOTE 61

Table 1 Isolates of Melampsora spp. used in the study. Pathotypes of Melampsora larici-populina determined as described in Pinon \& Frey (2005)

\begin{tabular}{|c|c|c|c|c|c|}
\hline Species & Isolate code & Pathotype & Original host & Collection site & Year \\
\hline \multicolumn{6}{|l|}{ Melampsora larici-populina } \\
\hline \multirow[t]{10}{*}{ World subpanel } & 97A1 & 0 & Populus $\times$ euramericana & Morocco & 1997 \\
\hline & 97A3 & $3-4$ & P. nigra & New Zealand & 1997 \\
\hline & 97J10 & $3-4$ & P. $\times$ euramericana 'I- $488^{\prime}$ & South Africa & 1997 \\
\hline & 97EA2 & 4 & Populus sp. & China & 1997 \\
\hline & 97C3 & $1-3-4-5-6-7$ & P. $\times$ interamericana 'Beaupré' & UK & 1997 \\
\hline & 98AE3 & 2 & Populus sp. & Finland & 1998 \\
\hline & 99D1 & $3-4$ & P. trichocarpa & Iceland & 1999 \\
\hline & 00A19 & 0 & P. $\times$ euramericana 'I- $488^{\prime}$ & Chile & 2000 \\
\hline & 98AR1 & $1-3-4-5-7-8$ & P. $\times$ interamericana 'B-71085/A1' & Belgium & 1998 \\
\hline & 95US1 & $3-4$ & P. nigra 'Italica' & USA & 1995 \\
\hline \multirow[t]{10}{*}{ Regional subpanel } & $94 Z Z 5$ & $1-3-4-5-7$ & P. $\times$ euramericana 'Ghoy' & N France & 1994 \\
\hline & 95AAH12 & $1-3-4-5-6-7$ & P. $\times$ interamericana 'Beaupré' & NE France & 1995 \\
\hline & 95XP5 & $1-3-4-5-6-7$ & P. $\times$ interamericana 'Beaupré' & SW France & 1995 \\
\hline & 95XW2 & $1-3-4-5-7$ & P. $\times$ interamericana 'Boelare' & W France & 1995 \\
\hline & 98AG31 & $3-4-7$ & P. $\times$ interamericana 'Beaupré' & N France & 1998 \\
\hline & $98 \mathrm{AO} 2$ & $3-4-7$ & P. $\times$ interamericana 'Beaupré' & SW France & 1998 \\
\hline & 00M10 & $3-4-7-8$ & P. $\times$ interamericana '69038- 1 ' & NE France & 2000 \\
\hline & $01 \mathrm{~L} 31$ & $2-3-7$ & P. $\times$ euramericana 'A4A' & C France & 2001 \\
\hline & 01L66 & $1-2-3-4-5-7$ & P. $\times$ euramericana 'Laxo 7' & NE France & 2001 \\
\hline & 02AO33 & 4 & P. nigra & SE France & 2002 \\
\hline \multirow[t]{11}{*}{ Local subpanel } & 991006 & $3-4$ & P. $\times$ euramericana 'Robusta' & NE France & 1999 \\
\hline & 991009 & $2-4$ & P. $\times$ euramericana 'Robusta' & NE France & 1999 \\
\hline & 991036 & $3-4-7$ & P. $\times$ euramericana 'Robusta' & NE France & 1999 \\
\hline & 991046 & $2-3-4$ & P. $\times$ euramericana 'Robusta' & NE France & 1999 \\
\hline & 991056 & $1-4-5$ & P. $\times$ euramericana 'Robusta' & NE France & 1999 \\
\hline & 991061 & $1-3-4-5-6$ & P. $\times$ euramericana 'Robusta' & NE France & 1999 \\
\hline & 991094 & $1-2-3-4-5-7$ & P. $\times$ euramericana 'Robusta' & NE France & 1999 \\
\hline & 99I108 & 4 & P. $\times$ euramericana 'Robusta' & NE France & 1999 \\
\hline & 991179 & $3-4-5$ & P. $\times$ euramericana 'Robusta' & NE France & 1999 \\
\hline & $99 I 192$ & $4-7$ & P. $\times$ euramericana 'Robusta' & NE France & 1999 \\
\hline & 93ID6 & $3-4$ & P. $\times$ euramericana 'I 45-51' & NE France & 1993 \\
\hline \multirow[t]{16}{*}{ Melampsora allii-populina } & $98 Z 1$ & - & Arum sp. & SE France & 1998 \\
\hline & $98 Z 3$ & - & Allium sp. & SE France & 1998 \\
\hline & $98 Z 4$ & - & Allium sp. & SE France & 1998 \\
\hline & $98 Z 5$ & - & Allium sp. & SE France & 1998 \\
\hline & $98 Z 6$ & - & Arum sp. & SE France & 1998 \\
\hline & $98 Z 9$ & - & Muscari comosum & SE France & 1998 \\
\hline & $98 Z 10$ & - & Allium porrum & SE France & 1998 \\
\hline & 89A1 & - & P. $\times$ euramericana 'Altichiero' & SW France & 1989 \\
\hline & 96B6-1 & - & Allium vineale & NE France & 1996 \\
\hline & $96 \mathrm{M} 24-2$ & - & Muscari comosum & NE France & 1996 \\
\hline & 97M2 & - & Allium sphaerocephalum & NE France & 1997 \\
\hline & $97 \mathrm{~N} 1$ & - & Arum maculatum & NE France & 1997 \\
\hline & 94HY8 & - & P.nigra & SE France & 1994 \\
\hline & 94IV7 & - & P. nigra & SE France & 1994 \\
\hline & $91 \mathrm{E} 4$ & - & P. $\times$ euramericana 'Robusta' & W France & 1991 \\
\hline & 91L5 & - & P. $\times$ interamericana 'Beaupré' & W France & 1991 \\
\hline Melampsora medusae & 88MM2 & - & P. $\times$ interamericana 'Beaupré' & SW France & 1988 \\
\hline \multirow{6}{*}{ f. sp. deltoidae } & 97CN1 & - & P. $\times$ interamericana 'Boelare' & SW France & 1997 \\
\hline & 98B1 & - & P. $\times$ interamericana ' $87002-21^{\prime}$ & South Africa & 1998 \\
\hline & 98D1 & - & P. $\times$ euramericana ' $5006^{\prime}$ & South Africa & 1998 \\
\hline & 99A3 & - & P. $\times$ interamericana 'Hoogvorst' & SW France & 1999 \\
\hline & 99T2 & - & P. $\times$ interamericana 'Hazendans' & NE France & 1999 \\
\hline & $01 Z 1$ & - & P. deltoides & Canada & 2001 \\
\hline
\end{tabular}


Table 1 Continued

\begin{tabular}{|c|c|c|c|c|c|}
\hline Species & Isolate code & Pathotype & Original host & Collection site & Year \\
\hline & $01 Z 2$ & - & P. deltoides & Canada & 2001 \\
\hline & $01 Z 4$ & - & P. deltoides & Canada & 2001 \\
\hline & $01 Z 5$ & - & P. deltoides & Canada & 2001 \\
\hline & $01 Z 11$ & - & P. $\times$ interamericana 'Unal' & Canada & 2001 \\
\hline & $02 \mathrm{AZ} 5$ & - & P. deltoides & Canada & 2002 \\
\hline & 02AZ6 & - & P. deltoides & Canada & 2002 \\
\hline & 02AZ7 & - & P. deltoides & Canada & 2002 \\
\hline & 02AZ8 & - & P. deltoides & Canada & 2002 \\
\hline & 02AZ11 & - & $P . \times$ jackii & Canada & 2002 \\
\hline Melampsora larici-tremulae & 01F1 & - & P.tremula & NE France & 2001 \\
\hline Melampsora rostrupii & $01 \mathrm{G} 1$ & - & Mercurialis perennis & NE France & 2001 \\
\hline \multirow[t]{3}{*}{ Melampsora pinitorqua } & 00S1 & - & P.tremula & SW France & 2000 \\
\hline & $00 S 2$ & - & P.tremula & S France & 2000 \\
\hline & $00 S 3$ & - & P.tremula & C France & 2000 \\
\hline
\end{tabular}

on a panel of $30 \mathrm{M}$. larici-populina isolates comprising three nested geographical (world, regional, local) subpanels with high pathotype diversity (Table 1 ).

PCR was performed using a PTC-200 Peltier Thermal Cycler (MJ Research) with $5 \mathrm{~min}$ at $95^{\circ} \mathrm{C}$ followed by 40 cycles of $60 \mathrm{~s}$ at $94{ }^{\circ} \mathrm{C}, 90 \mathrm{~s}$ at annealing temperature and $60 \mathrm{~s}$ at $72{ }^{\circ} \mathrm{C}$ and a final extension step of $30 \mathrm{~min}$ at $60^{\circ} \mathrm{C}$. PCR was carried out in $20 \mu \mathrm{L}$ final reaction volumes containing $15 \mathrm{ng}$ template DNA, $2 \mu \mathrm{L}$ of $10 \times$ reaction buffer, $1.5 \mathrm{~mm}$ $\mathrm{MgCl}_{2}, 0.2 \mathrm{~mm} \mathrm{dNTP}, 0.5 \mathrm{U}$ Taq polymerase (Sigma) and $0.2 \mu \mathrm{M}$ forward and reverse primers. Out of 37 loci tested, 22 failed to have an interpretable pattern of migration on a $2 \%$ agarose gel because of multiple bands or unexpected allele size. Forward primers of the remaining 15 loci (Table 2) were labelled to allow size and dye multiplexing. PCR products were separated, sized and analysed on a CEQ 8000 Genetic Analysis System (Beckman Coulter).

Out of 15 loci tested, 13 were found to be polymorphic with three to eight alleles (Table 2). Expected and observed heterozygosities as well as a test for Hardy-Weinberg expectation were computed using GENEPOP 3.4 (Raymond \& Rousset 1995). Departure from expected results was significant for eight loci, which could be explained by either a nonequilibrium state of the population, the sampling structure or a low frequency of null alleles. Locus $\mu \mathrm{MLP} 22$ was suspected to be uniparentally inherited because of the total lack of heterozygotes within the panel. The sequence adjacent to this locus was shown to exhibit $56 \%$ identity (29/51 amino acids) with the $5^{\prime}$ end of the cytochrome $c$ oxydase subunit 2 gene of the mitochondrion of the Basidiomycete Crinipellis perniciosa using NCBI's BLASTX program (Altschul et al. 1997), strongly suggesting that $\mu$ MLP22 was a mitochondrial locus. All other loci did not show any significant homology with nucleotide or protein databases.
Loci $\mu$ MLP12 and $\mu$ MLP13 were isolated from the same clone sequence and are thus physically linked, with a 90-bp interval between the two microsatellite motifs. Nevertheless, no significant linkage disequilibrium $(P<0.01)$ was detected among all pairs of loci for the 30 isolates of the test panel, according to an exact test performed with GENEPOP 3.4.

All loci were tested for cross-species amplification against M. allii-populina (16 isolates), M. medusae f. sp. deltoidae (16 isolates), M. larici-tremulae (1 isolate), M. rostrupii (1 isolate) and $M$. pinitorqua (3 isolates). Six loci resulted in global or partial amplification with some related species, but very few intraspecific polymorphisms were detected (Table 2).

\section{Acknowledgements}

We thank Christine Delaruelle, Annegret Kohler, Céline Lalanne, Patrick Léger and Martina Peter for their advice and help. This work was partly funded by grants from GIP ECOFOR and from INRA.

\section{References}

Altschul SF, Madden TL, Schäffer AA et al. (1997) Gapped BLAST and PSI-BLAST: a new generation of protein database search programs. Nucleic Acids Research, 25, 3389-3402.

Dutech C, Amsellem L, Billotte N, Jarne P (2000) Characterization of (GA) ${ }_{n}$ microsatellite loci using an enrichment protocol in the neotropical tree species Vouacapoua americana. Molecular Ecology, 9, 1433-1435.

Edwards KJ, Barker JHA, Daly A, Jones C, Karp A (1996) Microsatellite libraries enriched for several microsatellite sequences in plants. BioTechniques, 20, 758-760.

Enjalbert J, Duan X, Giraud T et al. (2002) Isolation of twelve microsatellite loci, using an enrichment protocol, in the phytopathogenic fungus Puccinia striiformis f. sp. tritici. Molecular Ecology Notes, 2, 563-565.

Estoup A, Solignac M, Harry M, Cornuet JM (1993) Characterization of $(\mathrm{GT})_{n}$ and $(\mathrm{CT})_{n}$ microsatellites in two insect species: Apis 


\begin{tabular}{|c|c|c|c|c|c|c|c|c|c|c|c|c|c|}
\hline Locus & $\begin{array}{l}\text { GenBank } \\
\text { Accession nos }\end{array}$ & Primer sequences $\left(5^{\prime}-3^{\prime}\right)$ & Repeat motif of cloned allele & $\begin{array}{l}\text { Expected } \\
\text { size (bp) }\end{array}$ & $\begin{array}{l}T_{\mathrm{a}} \\
\left({ }^{\circ} \mathrm{C}\right)\end{array}$ & Number of alleles (size in bp) & $H_{\mathrm{E}}$ & $H_{\mathrm{O}}$ & $\begin{array}{l}\text { Melampsora } \\
\text { alli-populina }\end{array}$ & $\begin{array}{l}\text { Melampsora } \\
\text { medusae } \\
\text { f. sp. deltoidae }\end{array}$ & $\begin{array}{l}\text { Melampsora } \\
\text { larici-tremulae }\end{array}$ & $\begin{array}{l}\text { Melampsora } \\
\text { rostrupii }\end{array}$ & $\begin{array}{l}\text { Melampsora } \\
\text { pinitorqua }\end{array}$ \\
\hline$\mu$ MLP09 & DQ059602 & $\begin{array}{l}\text { F1 TCAAGAACCAAACCAATCAAAC } \\
\text { R1 CATGCCAAACCATACTCCTG }\end{array}$ & $(\mathrm{CA})_{2} \mathrm{C}(\mathrm{CA})_{5} \mathrm{~A}(\mathrm{CA})_{5}$ & 171 & 60 & $\begin{array}{l}4 \\
(159,165,167,169)\end{array}$ & 0.40 & 0.47 & $0 / 16$ & $0 / 16$ & $0 / 1$ & $0 / 1$ & $0 / 3$ \\
\hline$\mu \mathrm{MLP} 12$ & DQ059603 & $\begin{array}{l}\text { F1 TGCTTCACAAACAAGACGAAAG } \\
\text { R1 ACCTTTGTCGACCAACAACC }\end{array}$ & $\begin{array}{l}(\mathrm{AAG})_{5}(\ldots)(\mathrm{AAG})_{6}(\ldots) \\
(\mathrm{AAG})_{10}(\ldots)(\mathrm{ATG})_{3}(\mathrm{AAG})_{4}\end{array}$ & 245 & 60 & $\begin{array}{l}7 \\
(239,242,245,247,248,250,266)\end{array}$ & 0.70 & 0.63 & $0 / 16$ & $0 / 16$ & $0 / 1$ & $0 / 1$ & $0 / 3$ \\
\hline$\mu \mathrm{MLP13}$ & DQ059604 & $\begin{array}{l}\text { F1 GTTGTTGGTCGACAAAGGTG } \\
\text { R1 GCATAAAGCCGCTGATTTTC }\end{array}$ & $(\mathrm{TG})_{11}$ & 187 & 60 & $\begin{array}{l}4 \\
(189,191,193,203)\end{array}$ & 0.45 & 0.47 & $0 / 16$ & $0 / 16$ & $0 / 1$ & $0 / 1$ & $0 / 3$ \\
\hline$\mu \mathrm{MLP} 20$ & DQ059605 & $\begin{array}{l}\text { F1 CTTTCAAGCTCAATGCAAGG } \\
\text { R1 AAACCAAGCAAGCATACCAC }\end{array}$ & $(\mathrm{TG})_{10}$ & 181 & 60 & $\begin{array}{l}3 \\
(177,179,181)\end{array}$ & 0.07 & 0.07 & $0 / 16$ & $0 / 16$ & $0 / 1$ & $0 / 1$ & $0 / 3$ \\
\hline $\mu$ MLP22 2 & DQ059606 & $\begin{array}{l}\text { F1 CGCATTCTATTTATTTAAACACACG } \\
\text { R1 CGATTCTGATCCATACCTGTCC }\end{array}$ & $(\mathrm{TA})_{8}(\ldots)(\mathrm{TAATAG})_{11}(\ldots)(\mathrm{TG})_{7}$ & 315 & 60 & $\begin{array}{l}4 \\
(312,318,324,330)\end{array}$ & 0.62 & $0.00^{*}$ & $\begin{array}{l}16 / 16 \\
280 \mathrm{bp}\end{array}$ & $\begin{array}{r}16 / 16 \\
291 \mathrm{bp}\end{array}$ & $0 / 1$ & $\begin{array}{r}1 / 1 \\
274 \mathrm{bp}\end{array}$ & $0 / 3$ \\
\hline$\mu \mathrm{MLP} 24$ & DQ059607 & $\begin{array}{l}\text { F1 GATCACGCCCATGCTTTAAC } \\
\text { R1 ATTTCCGGAAGTCCTTGACG }\end{array}$ & $(\mathrm{TG})_{8}(\ldots)(\mathrm{TG})_{5}$ & 154 & 60 & $\begin{array}{l}1 \\
(156)\end{array}$ & 0.00 & 0.00 & $0 / 16$ & $0 / 16$ & $0 / 1$ & $0 / 1$ & $0 / 3$ \\
\hline$\mu \mathrm{MLP27}$ & DQ059608 & $\begin{array}{l}\text { F1 CATGCTAATTGTATTGAGCTGTATTG } \\
\text { R1 TGGATGAGGATGATGAGAGG }\end{array}$ & $(\mathrm{TC})_{9} \mathrm{TTT}(\mathrm{TC})_{9}$ & 250 & 60 & $\begin{array}{l}4 \\
(240,242,250,252)\end{array}$ & 0.49 & $0.13^{*}$ & $\begin{array}{l}13 / 16 \\
228-230 \text { bp }\end{array}$ & $0 / 16$ & $0 / 1$ & $1 / 1$ & $0 / 3$ \\
\hline$\mu$ MLP28 & DQ059609 & $\begin{array}{l}\text { F1 ATCCCATGGAATCCGAAATG } \\
\text { R1 GCTGATTGAGCTCGGAGTTG }\end{array}$ & $(\mathrm{CACCA})_{6}(\ldots)(\mathrm{CACCA})_{3}$ & 459 & 60 & $\begin{array}{l}6 \\
(395,434,439,444,459,469)\end{array}$ & 0.29 & $0.21^{*}$ & $0 / 16$ & $0 / 16$ & $0 / 1$ & $0 / 1$ & $\begin{array}{c}3 / 3 \\
317-347 \mathrm{bp}\end{array}$ \\
\hline بMLP29 & DQ059610 & $\begin{array}{l}\text { F1 TGGACAAGTCCAAAATCATCAC } \\
\text { R1 GCTCGGAGTTGTGGTAGTGAG }\end{array}$ & $(\mathrm{CACCA})_{4}$ & 298 & 60 & $\begin{array}{l}3 \\
(295,300,310)\end{array}$ & 0.07 & $0.04^{*}$ & $0 / 16$ & $0 / 16$ & $0 / 1$ & $0 / 1$ & $0 / 3$ \\
\hline$\mu \mathrm{MLP} 30$ & DQ059611 & $\begin{array}{l}\text { F1 TGATGGGTTAGATGATGATTCC } \\
\text { R1 CAACACACAACAACACACAATTC }\end{array}$ & $\begin{array}{l}(\text { TGTGTGT })_{5}(\ldots) \\
(\text { TGTGTGT) }(. . .)(\text { TGTGTGT) }\end{array}$ & 249 & 63 & $\begin{array}{l}5 \\
(247,249,256,263,314)\end{array}$ & 0.25 & $0.13^{*}$ & $0 / 16$ & $0 / 16$ & $0 / 1$ & $0 / 1$ & $0 / 3$ \\
\hline$\mu \mathrm{MLP} 31$ & DQ059612 & $\begin{array}{l}\text { F1 CCACGACAGAGACGATATAGTTG } \\
\text { R1 CTTTCCCACACTTGTTTTGC }\end{array}$ & $(\mathrm{GT})_{3} \mathrm{GCTG}(\mathrm{GT})_{9}$ & 194 & 54 & $\begin{array}{l}6 \\
(182,183,184,187,190,192)\end{array}$ & 0.22 & 0.20 & $0 / 16$ & $0 / 16$ & $0 / 1$ & $0 / 1$ & $0 / 3$ \\
\hline$\mu$ MLP32 & DQ059613 & $\begin{array}{l}\text { F2 CGGATGCCTTAAGACTGTGG } \\
\text { R1 AAAGCCAACAAAGATGACCTG }\end{array}$ & $(\mathrm{TC})_{18}$ & 132 & 60 & $\begin{array}{l}8 \\
(116,122,124,126,128,136,138,140)\end{array}$ & 0.82 & $0.93^{*}$ & $0 / 16$ & $\begin{array}{r}1 / 16 \\
136 \mathrm{bp}\end{array}$ & $\begin{array}{r}1 / 1 \\
118 \mathrm{bp}\end{array}$ & $0 / 1$ & $\begin{array}{r}2 / 3 \\
118 \mathrm{bp}\end{array}$ \\
\hline$\mu \mathrm{MLP} 34$ & DQ059614 & $\begin{array}{l}\text { F3 GAGAAATCGAACGCCAGAAG } \\
\text { R2 TCAAAAGAATTGGGGACTGG }\end{array}$ & $(\mathrm{AG})_{13} \mathrm{AA}(\mathrm{AG})_{8}$ & 298 & 51 & $\begin{array}{l}1 \\
(268)\end{array}$ & 0.00 & 0.00 & $0 / 16$ & $0 / 16$ & $0 / 1$ & $0 / 1$ & $0 / 3$ \\
\hline$\mu \mathrm{MLP} 36$ & DQ059615 & $\begin{array}{l}\text { F1 TTTGAAAAAGTAATCGGAAGTGTG } \\
\text { R1 TCAGGTTAGCTTTTCGTTGG }\end{array}$ & $(\mathrm{AG})_{14}$ & 240 & 50 & $\begin{array}{l}6 \\
(226,228,230,232,236,238)\end{array}$ & 0.59 & $0.57^{*}$ & $0 / 16$ & $0 / 16$ & $0 / 1$ & $\begin{array}{c}1 / 1 \\
228-236 \mathrm{bp}\end{array}$ & $0 / 3$ \\
\hline$\mu \mathrm{MLP37}$ & DQ059616 & $\begin{array}{l}\text { F1 CCACGGTTGTGAAGTTGTATTC } \\
\text { R1 ACTTTTGACGTTCGATCACC }\end{array}$ & $(\mathrm{TC})_{10}$ & 224 & 60 & $\begin{array}{l}3 \\
(222,224,226)\end{array}$ & 0.50 & $0.63^{*}$ & $0 / 16$ & $0 / 16$ & $\begin{array}{c}1 / 1 \\
223-229 \mathrm{bp}\end{array}$ & $0 / 1$ & $\begin{array}{c}2 / 3 \\
225-231 \mathrm{bp}\end{array}$ \\
\hline
\end{tabular}




\section{PRIMER NOTE}

mellifera and Bombus terrestris. Nucleic Acids Research, 21, 14271431.

Frey P, Gérard P, Feau N, Husson C, Pinon J (2005) Variability and population biology of Melampsora rusts on poplars. In: Rust Diseases of Willow and Poplar (eds Pei MH, McCracken AR), pp. 63-72. CAB International, Wallingford, UK.

Lim S, Notley-McRobb L, Lim M, Carter DA (2004) A comparison of the nature and abundance of microsatellites in 14 fungal genomes. Fungal Genetics and Biology, 41, 1025-1036.

Pei MH, Whelan MJ, Halford NG, Royle DJ (1997) Distinction between stem- and leaf-infecting forms of Melampsora rust on Salix viminalis using RAPD markers. Mycological Research, 101, 7-10.
Pinon J, Frey P (2005) Interactions between poplar clones and Melampsora populations and their implications for breeding for durable resistance. In: Rust Diseases of Willow and Poplar (eds Pei MH, McCracken AR), pp. 139-154. CAB International, Wallingford, UK.

Raymond M, Rousset F (1995) GENEPOP (version 1.2): population genetics software for exact tests and ecumenicism. Journal of Heredity, 86, 248-249.

Rozen S, Skaletsky H (2000) PRIMER 3 on the WWW for general users and for biologist programmers. In: Bioinformatics Methods and Protocols: Methods in Molecular Biology (eds Krawetz S, Misener S), pp. 365-386. Humana Press, Totowa, New Jersey. 